M.C.M. Wong ${ }^{1}$, E.C.M. Lo ${ }^{1 *}$, E. Schwarz ${ }^{1,3}$, and H.G. Zhang ${ }^{2}$

${ }^{1}$ Faculty of Dentistry, The University of Hong Kong, 3/F, Prince Philip Dental Hospital, 34 Hospital Road, Hong Kong; and ${ }^{2}$ Department of Preventive Dentistry, Sun YatSen University of Medical Sciences; ${ }^{3}$ present address, 1619 Duke St., Alexandria, VA 22314, USA; * corresponding author,hrdplcm@hkucc.hku.hk

J Dent Res 80(5):1459-1465, 2001

\section{ABSTRACT}

The objectives of the study were to describe the oral health status and treatment needs of the 5- to 6-yearold and 12-year-old children in Southern China; to describe the patterns of oral health behaviors, knowledge, and attitudes among the 12-year-olds; and to assess the effects of socio-behavioral factors on the 12-year-old children's dental caries experiences. The study sample was comprised of 15875 - to 6-year-old and 1576 12-year-old urban and rual schoolchildren living in Guangdong Province. Three calibrated dentists clinically examined the children, and trained interviewers interviewed the 12year-olds. Caries prevalence of the 5- to 6-year-old children was high (urban $78 \%$ vs. rural $86 \%$ ); the mean dmft of the urban and rural children was 4.8 and 7.0, respectively. The caries prevalence and mean DMFT score of the 12-year-olds were $41 \%$ and 0.9 (urban) and $42 \%$ and 0.9 (rural). Only $2 \%$ of the $12-$ year-olds exhibited no calculus or gingival bleeding, while more than $70 \%$ had calculus. In conclusion, there is an urgent need for establishing cariespreventive activities for preschool children. The prevalence of caries among the 12-year-olds was not high, but their periodontal condition was unsatisfactory. Knowledge about gum bleeding and the use of fluoride was low. More oral health education activities should be organized, especially for the rural children.

KEY WORDS: child, caries, CPI, oral health behavior, Chinese.

The Oral Health Survey in Southern China, 1997, was conducted under the auspices of the Department of Periodontology and Public Health of the Faculty of Dentistry, the University of Hong Kong, and publication of these papers is made possible by funding from The Research Grants Council of the Hong Kong Special Administrative Region, China, HKU 232/95M, together with the University of Hong Kong Committee for Research and Conference Grants.

A supplemental appendix to this article is published electronically only at http://www.dentalresearch.org.

\title{
Oral Health Status and Oral Health Behaviors in Chinese Children
}

\section{INTRODUCTION}

D ental caries prevalence in pre-school children has declined in most of the highly industrialized countries over the last two decades but is still high in the developing countries (Holm, 1990; Fejerskov et al., 1994; Murray, 1994). One of the goals set by the World Health Organization (1988) for oral health was that by the year $2000,50 \%$ of the 5- to 6-year-olds should be caries-free. Most of the previous oral health surveys conducted in China were on children and adolescents. In the First National Oral Health Survey, conducted in 1983, it was found that the mean $\mathrm{dmft}$ scores of the 7-year-old children were 4.8 in urban areas and 2.6 in rural areas, with caries prevalences of $84 \%$ and $62 \%$, respectively (Ministry of Public Health, 1987). In Guangdong Province, the caries situation of urban children was similar to, but that of rural children was higher than, that reported at the national level (Zheng et al., 1986). Two recent oral epidemiological surveys conducted in selected areas in Guangdong Province in 1987 and 1992 showed that the caries prevalence among pre-school children was from 80 to $90 \%$ (Wang et al., 1994; Wong et al., 1997), which was far from the oral health goal set by the World Health Organization (WHO).

Prevalence of dental caries for the schoolchildren also varied greatly between different provinces in China. For instance, in the 1970s, the caries prevalence of schoolchildren ranged from $13 \%$ in Guiyang Province to $81 \%$ in Haerbin Province (Yue, 1980; Editorial Board of Chinese Journal of Stomatology, 1983; Editorial Board of West China Journal of Stomatology, 1988). The First National Oral Health Survey reported that the prevalence of dental caries among urban 12-year-olds was $46 \%$ and the mean DMFT score was 1.0, while the corresponding figures for rural 12 -year-olds were $31 \%$ and 0.7 (Ministry of Public Health, 1987). Results from more recent surveys showed that the mean DMFT scores of 12-year-olds ranged from 0.2 to 1.9 in different provinces in China (Hu and Liu, 1992; Shi et al., 1992; Li et al., 1994; Petersen and Guang, 1994; Peng et al., 1997; Feng et al., 1998; Petersen and Esheng, 1998; Lo et al., 1999). Surveys have also shown that the gingival health status of Chinese children was unsatisfactory (Wei et al., 1986; Wright et al., 1989; Peng et al., 1997). However, the current dental caries prevalence and gingival health status of 12-year-olds in Guangdong Province in Southern China are unknown. In a survey conducted in neighboring Hong Kong in 1995, the mean DMFT score of 12-year-olds was found to be 1.0 (Chan et al., 1997).

It is now well-known that systemically administered tetracycline can cause discoloration in primary and permanent teeth (Mello, 1967; Pindborg, 1982; Warnakulasuriya, 1989). However, in the 1960s and ' $70 \mathrm{~s}$, the use of tetracycline as an antibiotic for common medical problems in children was quite popular in China. In the First National Oral Health Survey (Ministry of Public Health, 1987), the prevalence of tetracycline-stained teeth was found to be high among schoolchildren $(23 \%)$. Although the Chinese government has banned the use of tetracycline among children since the 1980s (Editorial Board of Chinese Annals of Public Health, 1983), information on the prevalence of tetracycline staining in the primary and permanent teeth of Chinese children after the implementation of this regulation was lacking. 


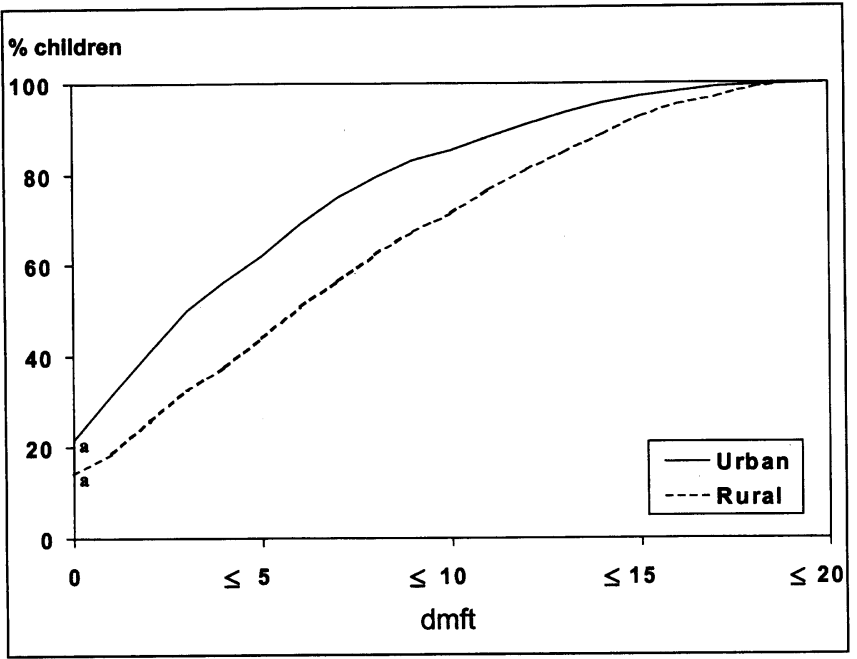

Figure 1. Cumulative distribution of dmft score among the 5- to 6-yearold urban and rural children. "Significant difference in the prevalence of caries between urban and rural children $(p<0.001)$.

Unlike oral health status, the oral health behaviors, knowledge, and attitudes of children were seldom studied in China. Since the late 1980 s, the health authorities have emphasized preventive oral care and health education to improve the oral health status and behavior of the public (Peng et al., 1997). However, without information about oral health behaviors, knowledge, and attitudes of the children, it would be difficult to plan and conduct educational activities which tailor messages and target behaviors that need to be changed.

The objectives of the present analysis were: first, to describe the oral health status, including dental caries, prevalence of tetracycline-stained teeth, and treatment needs, of the 5- to 6-year-

Table 1. Dental Caries Prevalence and Experience of the Surveyed Children According to Gender and Location of Residence (standard error in parentheses)

\begin{tabular}{|c|c|c|c|c|c|c|}
\hline & $n$ & $\% \mathrm{dmft}>0$ & $d t$ & $\mathrm{mt}$ & ft & $\mathrm{dmft}$ (SE) \\
\hline \multicolumn{7}{|c|}{$\begin{array}{l}\text { 5- to 6-year-olds } \\
\text { Urban }\end{array}$} \\
\hline Boy & 406 & 78 & 4.8 & $<0.1$ & 0.1 & $5.0(0.2)$ \\
\hline Girl & 396 & 79 & 4.5 & $<0.1$ & 0.1 & $4.7(0.2)$ \\
\hline Total & 802 & 78 & 4.7 & $<0.1$ & 0.1 & $4.8(0.2)^{\mathrm{a}}$ \\
\hline \multicolumn{7}{|l|}{ Rural } \\
\hline Boy & 405 & $90^{\mathrm{b}}$ & 7.7 & $<0.1$ & $<0.1$ & $7.7(0.3)^{b}$ \\
\hline Girl & 380 & $82^{b}$ & 6.3 & $<0.1$ & $<0.1$ & $6.4(0.3)^{b}$ \\
\hline \multirow[t]{2}{*}{ Total } & 785 & 86 & 7.0 & $<0.1$ & $<0.1$ & $7.0(0.2)^{\mathrm{a}}$ \\
\hline & $n$ & $\%$ DMFT > 0 & DT & MT & FT & DMFT (SE) \\
\hline \multicolumn{7}{|c|}{ 12-year-olds } \\
\hline \multicolumn{7}{|c|}{ Urban } \\
\hline Boy & 406 & 41 & 0.7 & $<0.1$ & 0.1 & $0.8(0.06)$ \\
\hline Girl & 388 & 41 & 0.7 & $<0.1$ & 0.2 & $0.9(0.08)$ \\
\hline Total & 794 & 41 & $0.7^{c}$ & $<0.1$ & $0.1^{a}$ & $0.9(0.05)$ \\
\hline \multicolumn{7}{|l|}{ Rural } \\
\hline Boy & 396 & 36 & 0.8 & $<0.1$ & $<0.1$ & $0.8(0.07)$ \\
\hline Girl & 386 & 48 & 1.0 & $<0.1$ & $<0.1$ & $1.1(0.08)$ \\
\hline Total & 782 & 42 & $0.9 c$ & $<0.1$ & $<0.1^{\circ}$ & $0.9(0.05)$ \\
\hline
\end{tabular}

a Significant difference between urban and rural children $(p<0.001)$.

b Significant difference between rural boys and girls ( $p<0.001$ ).

c Significant difference between urban and rural children $(p=0.010)$. olds, and the dental caries, periodontal conditions, prevalence of tetracycline-stained teeth and dental fluorosis, and treatment needs in the 12-year-olds in Southern China; second, to describe the patterns of oral health behaviors, knowledge, and attitudes among the 12-year-olds; and third, to assess the effects of socio-behavioral factors on the 12-year-old children's dental caries experience.

\section{MATERIALS \& METHODS Subjects}

Two groups of children were surveyed: 5- to 6-year-olds and 12-yearolds. A total of 1587 children aged 5 to 6 years was examined- -802 from urban areas ( 406 boys and 396 girls) and 785 from rural areas (405 boys and 380 girls). As for the 12-year-old age group, 1576 children were surveyed-794 from urban areas ( 406 boys and 388 girls) and 782 from rural areas ( 396 boys and 386 girls). Details of the survey protocol and the sampling procedures for the children have previously been described (Schwarz et al., 2001). In brief, a combination of multi-stage stratified sampling and quota sampling was used. The largest administrative regions - one each from the central, eastern, western, and northern parts of Guangdong Province - were chosen in the first stage. Then, by a two-stage stratified simple random sampling, 2 urban subdistricts and 2 rural townships in each region were selected. The 5- to 6year-old children were recruited from the largest kindergartens or primary schools (pre-school class) within the 16 sampling sites (8 urban and 8 rural) through the help of the Bureau of Education and Bureau of Public Health of the local government. Similarly, the 12-year-old children were recruited from the largest primary schools.

\section{Clinical Examination}

All children underwent a clinical examination which assessed their dental caries and treatment need at tooth level, their periodontal condition (12-year-olds only), the presence of dental fluorosis (12year-olds only), and the presence of tetracyclinestained teeth. The clinical criteria for the above diagnosis followed those recommended by the WHO (1997). The clinical examination took place under field conditions, with the use of plane mouth mirrors, WHO periodontal probes, and portable clinic lights. No radiographs were taken. Dental caries in the primary and permanent dentition was detected mainly visually at the cavitation level. Initial caries lesions were not recorded. The toothbased treatment needs included those relating to the removal of caries and restoration of lost tissue, pulp care, replacement of existing restorations, and tooth extraction. Assessment of the periodontal condition was carried out on the 6 index teeth according to the CPI index.

A photo album with photographs showing normal teeth, tetracycline-stained teeth, and teeth with various degrees of dental fluorosis was used in the calibration of examiners and also in the field to aid the diagnosis. For each child, tetracycline staining was recorded as either present or absent, while Dean's Index (WHO, 1997) was used to assess the degree of dental fluorosis.

Clinical examinations were carried out by the same three calibrated examiners throughout the survey. The survey took place over 2 periods of 2 months each, with a break of 2 months, and 2 administrative regions were surveyed in each 
period. Inter-examiner calibration was carried out before the start of each period. Duplicate examinations were conducted on one out of every ten children throughout the survey. Inter-examiner reliability on caries status at tooth level, as measured by Kappa statistics, was good ( 0.93 for both age groups), and inter-examiner reliability on the periodontal status, as measured by the weighted Kappa (0.60), was substantial.

\section{Questionnaire}

A structured questionnaire was used in this survey for the 12-yearold children, to gather information about their demographic background, oral health knowledge, attitudes and practices, dietary habits, and dental services utilization history. The children were interviewed by trained interviewers before they were clinically examined. Local health workers fluent in local dialects were recruited and trained as interviewers in this study, because some of the children could speak only their own local dialect.

Three scores were constructed to assess the dental knowledge, attitudes, and dietary habits of the children. Concerning dental knowledge, 4 questions were asked: the causes of dental caries and gum disease, and the ways to prevent dental caries and gum disease. For each question, up to 3 answers were recorded. A dental knowledge score was constructed by counting the total number of acceptable answers given by the children, excluding responses like 'do not know' and 'no answer'. Thus, the dental knowledge score was in a ratio scale and ranged from 0 to 12 (Schwarz and Lo, 1994; Lin et al., 2001), with a higher dental knowledge score indicating better dental knowledge.

Eight statements concerning oral health issues were made. The children were asked to indicate whether they agreed with, disagreed with, or had no comment on each of the statements. A dental attitude score was constructed by counting the total number of statements to which the children showed a positive attitude. Again, this score was in a ratio scale and ranged from 0 to 8 (Lin et al., 2001), with a higher score indicating a more positive attitude.

We chose to ask about 9 kinds of snack foods (with 5 of them being potentially highly cariogenic), to assess the dietary habits of the children. The children were asked how often they consumed the 9 kinds of snacks. A dietary habit score was constructed by counting the total number of potentially cariogenic snacks which the children consumed frequently (at least several times a week). Thus, the score was in a ratio scale and ranged from 0 to 5. The higher the score, the more frequent was the child's consumption of the potentially cariogenic snacks.

\section{Data Analyses}

DMFT/dmft scores were calculated for the surveyed children. We calculated the overall provincial mean DMFT/dmft score by weighting the DMFT/dmft scores obtained from the urban and rural samples according to the actual ratio of urban and rural residents in Guandong Province, i.e., 1:3 (Schwarz et al., 2001). The overall provincial prevalence of dental caries was calculated by the same approach. The differences in mean DMFT/dmft scores, dental knowledge scores (12-year-olds only), dental attitude scores (12-yearolds only), and dietary habit scores (12-year-olds only) between children who lived in urban and rural areas or between boys and girls were analyzed by two-sample $t$ tests. Differences in the prevalence of dental caries,

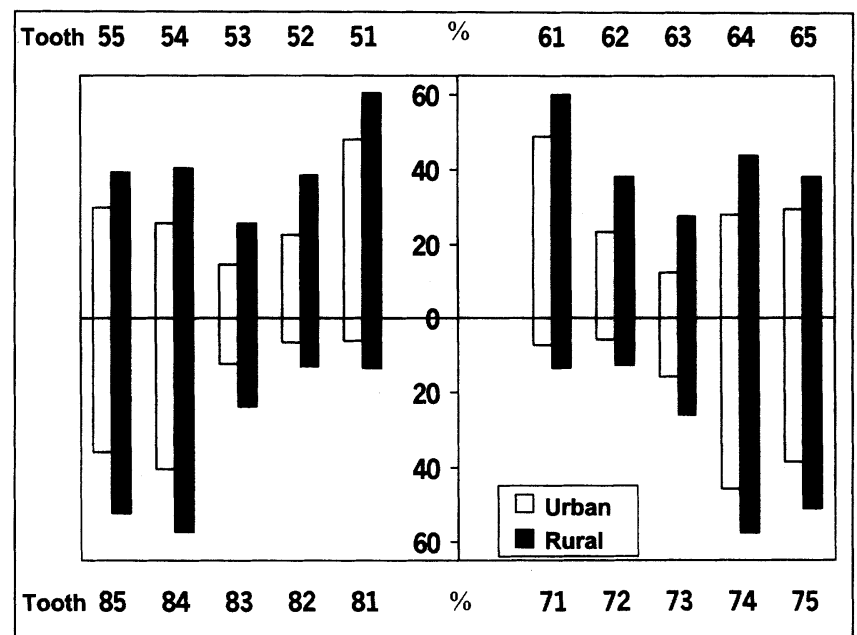

Figure 2. Dental caries attack rates in different types of primary teeth among the 5- to 6-year-old children.

dental fluorosis (12-year-olds only), prevalence of tetracyclinestained teeth, the distributions of periodontal conditions (12year-olds only), perceived oral health situation (12-year-olds only), oral health habits (12-year-olds only), and behaviors (12year-olds only) between groups were analyzed by Chi-squared tests. Based on the analytical model adopted in this study (Schwarz et al., 2001), we performed analysis of covariance (ANCOVA) to assess the effects of the predisposing, enabling, and behavioral factors on the DMFT scores of the 12-year-olds. The 8 selected variables were gender, location, frequency of toothbrushing, last dental visit, parents' education level, dental knowledge, dental attitude, and dietary habit scores. The level of statistical significance was set at 0.05 .

Table 2. Percentage of Surveyed Children Needing Treatment According to Gender, Location of Residence, and Treatment Type

\begin{tabular}{|c|c|c|c|c|c|}
\hline & $\begin{array}{c}\text { No } \\
\text { Treatment }\end{array}$ & $\begin{array}{l}\text { One-surface } \\
\text { Filling }\end{array}$ & $\begin{array}{l}\text { 2+-surface } \\
\text { Fillings }\end{array}$ & $\begin{array}{l}\text { Pulp Care } \\
\text { +Restoration }\end{array}$ & Extraction \\
\hline \multicolumn{6}{|c|}{$\begin{array}{l}\text { 5- to 6-year-olds } \\
\text { Urban }\end{array}$} \\
\hline Boy & 24 & 60 & 57 & 25 & 17 \\
\hline Girl & 23 & 62 & 56 & 25 & 12 \\
\hline Total & $23^{a}$ & $61^{a}$ & $56^{a}$ & $25^{a}$ & $14^{a}$ \\
\hline \multicolumn{6}{|l|}{ Rural } \\
\hline Boy & 12 & 75 & 74 & 46 & 36 \\
\hline Girl & 20 & 66 & 66 & 34 & 28 \\
\hline Total & $16^{a}$ & $71^{a}$ & $70^{a}$ & $40^{a}$ & $32^{a}$ \\
\hline \multicolumn{6}{|c|}{ 12-year-olds } \\
\hline \multicolumn{6}{|c|}{ Urban } \\
\hline Boy & 50 & 36 & 7 & 9 & 13 \\
\hline Girl & 57 & 31 & 6 & 9 & 11 \\
\hline Total & $54^{b}$ & 34 & $6^{b}$ & $9^{b}$ & $12^{b}$ \\
\hline \multicolumn{6}{|l|}{ Rural } \\
\hline Boy & 49 & 33 & 10 & 14 & 19 \\
\hline Girl & 44 & 42 & 11 & 14 & 16 \\
\hline Total & $47^{b}$ & 38 & $11^{b}$ & $14^{b}$ & $17 b$ \\
\hline
\end{tabular}

a Significant difference between urban and rural children $(p<0.001)$.

b Significant difference between urban and rural children $(p<0.01)$. 


\section{RESULTS}

\section{Oral Health Status}

The cumulative distributions of $\mathrm{dmft}$ for the 5- to 6-year-old urban children (children who lived in the urban areas) and rural children (children who lived in the rural areas) are illustrated in Fig. 1. It can be seen that the prevalence of dental caries was higher among the rural children than among the urban children, the respective percentages being $86 \%$ and $78 \%$ ( $p<0.001$ ). The overall provincial (weighted) prevalence of dental caries was estimated to be $84 \%$. Around $60 \%$ of the urban children had a dmft score smaller than 5 , and less than $20 \%$ of them had a dmft score greater than 10 . The corresponding percentages for the rural children were $40 \%$ and $30 \%$, respectively. The caries attack rates in different types of primary teeth are shown in Fig. 2 . It can be seen that the caries attack rates of the rural children were consistently higher than those of the urban children for each tooth type, although the pattern was similar in both groups.

The mean dmft score of the rural 5- to 6-year-old children was 7.0, while that of the urban children was 4.8 (Table 1). This difference was statistically significant $(\mathrm{p}<0.001)$. The overall provincial (weighted) mean $\mathrm{dmft}$ score was 6.5 . In the urban areas, the dental caries prevalences among boys and girls were similar. However, in the rural areas, the caries prevalence and caries experience of boys were higher than those of girls $(p<0.001)$. Nearly all of the recorded caries experience was decayed teeth.

The prevalence of dental caries and the mean DMFT scores were similar among the urban and the rural 12-year-old children (Table 1). The overall provincial (weighted) mean DMFT score and prevalence of dental caries were 0.9 and $42 \%$, respectively. Decayed teeth (DT) was the major component of the DMFT scores in both groups of children. However, the urban children had more filled teeth and fewer decayed teeth than the rural children $(p<0.01)$.

Only about one-quarter of the urban 5- to 6-year-old children and $16 \%$ of the rural children did not have any toothbased treatment need (Table 2). Since caries prevalence of rural children was higher than that of urban children, the proportions of rural children in need of treatment were higher than those of

Table 3. Percentages of 12-year-old Children with Tetracycline-stained Teeth and Dental Fluorosis According to Gender and Location of Residence

\begin{tabular}{|c|c|c|c|c|c|c|c|}
\hline & \multirow{2}{*}{$\begin{array}{l}\text { Tetracycline } \\
\text { Staining }\end{array}$} & \multicolumn{6}{|c|}{ Dean's Dental Fluorosis Index } \\
\hline & & Normal & Questionable & Very Mild & Mild & Moderate & Severe \\
\hline \multicolumn{8}{|l|}{ Urban } \\
\hline Boy & 2 & 83 & 11 & 3 & 2 & 1 & 0 \\
\hline Girl & 2 & 88 & 6 & 4 & 2 & 0 & 0 \\
\hline Totala & 2 & 85 & 8 & 4 & 2 & 1 & 0 \\
\hline \multicolumn{8}{|l|}{ Rural } \\
\hline Boy & 3 & 87 & 6 & 4 & 1 & 1 & 1 \\
\hline Girl & 4 & 91 & 4 & 3 & 1 & 0 & 1 \\
\hline Totala & 3 & 89 & 5 & 3 & 1 & 1 & 1 \\
\hline
\end{tabular}

a Significant difference in distribution of dental fluorosis between urban and rural children $(p=0.016)$. urban children in all treatment types. The need for fillings (either one-surface or multi-surface) was great in the surveyed children. Moreover, in more than one-third of the children, the caries lesions in some of their teeth were so advanced that these teeth were in need of pulp treatment or extraction.

About half of the 12-year-old children in all subgroups did not need any tooth-based treatment (Table 2). Most of the treatments needed were either one-surface or multi-surface fillings. Rural children had a bigger need for fillings, pulp care, and extractions than the urban children $(p<0.01)$.

The prevalence of tetracycline-stained teeth in the rural 5- to 6-year-old children was higher than that in the urban children $(3.7 \% v s .0 .5 \%)$, and this difference was statistically significant $(\mathrm{p}<0.001)$. However, the prevalence of tetracycline-stained teeth in boys was similar to that in girls.

Less than $5 \%$ of the surveyed 12-year-old children had the highest CPI score of zero, i.e., no gingival bleeding or calculus, and around three-quarters of the surveyed children were found to have calculus. When the CPI scores of the urban and rural children were compared, it was found that proportionally more urban children had healthy gums and fewer had calculus than the rural children $(p=0.021)$. No significant difference in the distribution of the CPI scores was found between boys and girls. Consistent with the above findings, on average, urban children were found to have more sextants assessed as healthy or bleeding only, and fewer sextants assessed as having calculus, than the rural children $(\mathrm{p}<0.01)$. No statistically significant difference in the mean numbers of sextants according to CPI scores was found between boys and girls. (The percentage distributions of children according to the highest CPI score and the mean number of sextants per child according to CPI scores are illustrated in Table A1 [electronic appendix].)

The prevalence of tetracycline-stained teeth was low among the surveyed 12-year-old children (Table 3 ). No statistically significant difference in prevalence was found between urban and rural children and between boys and girls. Less than $10 \%$ of the children in any subgroup showed signs of dental fluorosis beyond the questionable level (Table 3). The prevalence of dental fluorosis was higher among the urban children $(p=0.016)$, and, again, no significant difference was found between boys and girls.

\section{Perceived Oral Health, Knowledge, and Attitudes of 12-year-old Children}

When the children were asked to describe their perceived condition of their teeth and gums, $44 \%$ of the urban children answered "good", 45\% indicated "average", and 6\% indicated "poor", while $5 \%$ of the urban children had no idea. Proportionally fewer of the rural children thought their teeth and gums were good $(38 \%)$, and more thought their condition was poor $(15 \%)$ when compared with those of the urban children $(p<0.001)$, but no gender difference was found.

Among the urban children, 32\% were satisfied with the appearance of their teeth, $18 \%$ were not satisfied, and $50 \%$ had no comment. Proportionally more of the rural children were satisfied (43\%) with their teeth and fewer had no comment (33\%) when 
compared with the urban children ( $\mathrm{p}<0.001)$; again, no gender difference was found. Only $5 \%$ of the urban and $11 \%$ of the rural children avoided smiling or laughing because of their teeth, while $5 \%$ of the urban and $7 \%$ of the rural children had the experience of other schoolchildren making fun of their teeth.

The children's answers to the 4 questions on the causes and prevention of tooth decay and gum disease are shown in Table 4. Most of the children knew that consuming sugar and sweet foods could cause tooth decay. However, only $8 \%$ of the urban children and $5 \%$ of the rural children could identify bacteria and plaque as a cause of tooth decay, while $10-20 \%$ of the children had no idea. Concerning the causes of gum disease, around half of the children did not know (urban, 48\%; rural, 66\%). The main causes given by the children were poor oral hygiene, trauma from toothbrushing, and explanations according to Chinese health beliefs. Toothbrushing (urban, $81 \%$; rural, $48 \%$ ) and the use of fluoridated toothpaste (urban, 52\%; rural, 36\%) were the 2 most common answers given by the children when asked about ways to prevent tooth decay. Toothbrushing (urban, 24\%; rural, 14\%) was also the most common answer given for preventing gum disease. However, in fact, most of the children (urban, 49\%; rural, 71\%) did not know how to prevent gum disease. The mean dental knowledge scores of the urban and nural children were 4.8 and 3.1 , respectively $(p<0.001)$. The mean dental knowledge scores of the boys and girls were similar.

The dental attitudes of most children $(70 \%$ or more) were positive for most aspects, except for 2 issues. Only around half of the children agreed that tooth decay could make them look bad, while only $37 \%$ of the urban and $16 \%$ of the rural children agreed that using fluoride could be a good way to prevent tooth decay. The mean dental attitude scores were 6.2 and 5.4 for the urban children and the rural children, respectively $(\mathrm{p}<0.001)$. It was also found that boys had a higher dental attitude score than girls $(5.9$ vs. 5.6, $\mathrm{p}<0.001)$. (Supporting data are presented in Table A2 [electronic appendix].)

\section{Oral Health Behaviors of 12-year-old Children}

Among the urban children, $77 \%$ claimed that they brushed their teeth twice or more daily, and $22 \%$ of the children brushed their teeth once daily. A significantly lower $(p<0.001)$ percentage of the rural children claimed that they brushed their teeth twice or more daily $(31 \%)$, and proportionally more of them brushed only once a day $(67 \%)$. Furthermore, the reported toothbrushing habit was better among the girls (61\% twice or more daily) than among the boys $(48 \%$ twice or more daily, $\mathrm{p}<0.001)$. Almost all of the children $(99 \%)$ said that they used toothpaste when they brushed their teeth.

About half of the urban children had visited a dentist within the preceding year, while $13 \%$ of them had never visited a dentist before. The corresponding percentages for the rural children were $13 \%$ and $51 \%$, respectively. Thus, it can be seen that the rural children visited the dentist much less often than the urban children $(p<0.001)$. For the urban children who had visited a dentist, the main reasons for their visit were pain or troubles with their teeth $(35 \%)$, going for check-ups $(36 \%)$, and going for treatments $(27 \%)$. The main reasons for the rural children who had visited a dentist were pain or troubles with their teeth $(68 \%)$ and going for treatments $(20 \%)$. Treatments received during their last dental visit for the urban children were mainly dental examination (47\%), extraction (38\%), and fillings (15\%). For the rural children, main treatments received were extraction $(60 \%)$ and dental examination $(21 \%)$. Only $4 \%$ of the children had received fissure sealants.
Table 4. Responses of the 12-year-olds to the Questions on the Causes and Prevention of Tooth Decay and Gum Disease According to Location of Residence (percentages)

\begin{tabular}{cc}
$\begin{array}{c}\text { Urban } \\
(n=794)\end{array}$ & $\begin{array}{c}\text { Rural } \\
(n=782)\end{array}$ \\
\hline
\end{tabular}

Causes of tooth decay

Sugar and sweet food

Poor oral hygiene

Bacteria, plaque

Acid

Chinese explanations

"Tooth worm"a

Others

Do not know

$\begin{array}{rr}82 & 61 \\ 53 & 22 \\ 8 & 5 \\ 4 & 11 \\ 2 & 8 \\ 2 & 4 \\ 2 & 2 \\ 10 & 30\end{array}$

$\begin{array}{lrr}\text { Causes of gum disease } & & \\ \text { Poor oral hygiene } & 16 & 8 \\ \text { Trauma from toothbrushing } & 15 & 11 \\ \text { Chinese explanations } & 14 & 14 \\ \text { Bacteria, plaque } & 9 & 3 \\ \text { Inadequate diet, lack of vitamin C } & 4 & 1 \\ \text { Calculus } & 2 & 1 \\ \text { Others } & 8 & 5 \\ \text { Do not know } & 48 & 66\end{array}$

$\begin{array}{lcr}\text { Ways to prevent tooth decay } & & \\ \text { Toothbrushing } & 81 & 48 \\ \text { Take less sugar } & 52 & 36 \\ \text { Rinse mouth after eating } & 23 & 11 \\ \text { Visit a dentist } & 12 & 7 \\ \text { Use fluoridated toothpaste } & 4 & 1 \\ \text { Others } & 7 & 5 \\ \text { Do not know } & 7 & 33\end{array}$

Ways to prevent gum disease

$\begin{array}{lll}\text { Toothbrushing } & 24 & 14\end{array}$

Rinse mouth after eating $\quad 14 \quad 5$

Eat less hot food, drink cooling tea $\quad 11 \quad 9$

Visit a dentist

Use fluoridated toothpaste

Eat fruit, nourishing food

Others

Do not know

8
4
3
1
1
5
66

5

a Popular expression indicating an imaginary organism that causes dental decay.

Consumption of tea by the children was frequent. About $60 \%$ of the children drank tea daily, whereas the reported consumption of soft drinks was low: Only $9 \%$ of the children had soft drinks daily, and around $60 \%$ seldom or never had soft drinks. The reported consumption of sugary foods was also low, with $14 \%$ of the children saying that they had sweets/candy and $10 \%$ having cakes once daily, or more often, while around half of the children reported that they seldom or never had sweets/candy or cakes. The overall mean dietary habit score was 1.29 , and no statistically significant differences in the scores were found between urban and rural children, or between boys and girls. 


\section{Socio-Behavioral Factors and Oral Health in 12-year-old Children}

To investigate the effects of the socio-economic and behavioral factors on dental caries experience (i.e., DMFT score), we performed an ANCOVA. Among the 8 selected variables, only 3 remained in the final model (Table 5). It was found that being a girl, living in rural areas, and having visited a dentist within the preceding 3 years were associated with a higher mean DMFT score.

\section{DISCUSSION}

The 1983 Chinese national survey found that the caries prevalence was higher among the 7-year-old urban children than among the rural children (Ministry of Public Health, 1987). Contrary to this finding, the caries prevalence among the 5- to 6year-old rural children surveyed in the present study was higher than that among the urban children. Similar caries prevalence patterns were found in a recent study in Shanghai (Qiu et al., 1996). The reasons for this change might be due to: (1) the availability of fluoride toothpaste in the urban areas but not in the rural areas in recent years; (2) better oral health care for children in the urban areas; and (3) more oral health promotion and primary prevention programs being offered in urban areas. However, since the prevalence of dental caries was $86 \%$ for the rural 5- to 6-year-old children and 78\% for the urban children, this was still far from the oral health goal set by the WHO (1988), that $50 \%$ of 5- to 6-year-olds should be caries-free.

When the patterns of dental caries of the 5- to 6-year-old children were analyzed, it was found that caries was more prevalent on the upper incisors and molars. Similar patterns of dental caries among Chinese pre-school children had also been found in other studies (Douglass et al., 1995; Wong et al., 1997). However, inadequate information was sought in this and the other studies to investigate the etiology of caries among the pre-school children, e.g., whether it was due to the use of nursing bottles.

Results of a recent survey conducted in Hong Kong showed that caries prevalence in the 5-year-olds was $63 \%$ and the mean dmft score was 3.2 (Wei et al., 1993). These figures were lower than those found in this survey. The difference might be due to the fact that the domestic water in Hong Kong has been fluoridated since 1961 (Schwarz and Lo, 1995). Besides water fluoridation, fluoride toothpaste and other fluoride products are more readily available in Hong Kong.

When compared with the results of the First National Oral

Table 5. Results of ANCOVA with DMFT Value as the Dependent Variable in the 12-year-old Children

\begin{tabular}{llccc}
\hline Independent Variable & Estimate & SE (estimate) & p-value \\
\hline Location: & $\begin{array}{l}\text { Rural } \\
\text { Urban }\end{array}$ & 0.21 & 0.08 & 0.01 \\
Gender: & $\begin{array}{l}\text { Girl } \\
\text { Boy }\end{array}$ & 0.19 & 0.07 & 0.01 \\
Last dental visit: Within 3 yrs & 0.44 & 0.08 & $<0.01$ \\
(Intercept) & More than 3 yrs & 0.44 & 0.09 & $<0.01$ \\
\hline
\end{tabular}

a Reference category: $F=12.8 ; d f=3,1572 ; p<0.01$.
Health Survey conducted in 1983 (Ministry of Public Health, 1987), the present study showed that the prevalence of dental caries and mean DMFT score of the 12-year-old urban children in Guangdong Province had dropped, while those of the rural children remained unchanged. The findings of this study was similar to those of 2 recent surveys, one conducted in Shanghai (Feng et al., 1998) and one in Hubei Province, Central China (Peng et al., 1997). The present findings also showed that the dental caries experience of the 12-year-old Guangdong children was very similar to that of Hong Kong children (Chan et al., 1997). The only difference was that DT was the major component of the DMFT score of the children in this study, while FT was the major component among the 12-year-olds in Hong Kong. The main reason for this difference is probably the better accessibility of dental services in Hong Kong, since around $80 \%$ of the primary schoolchildren in Hong Kong join the government School Dental Care Service, which provides them with basic dental care services.

The periodontal health condition of the 12-year-olds surveyed was generally poor and was worse than that of 12year-olds in Central China (Peng et al., 1997) and in Hong Kong (Chan et al., 1997). This may be related to their poor knowledge of the causes of and ways to prevent gum disease, especially for the rural children. Moreover, since around half of the children claimed that they brushed their teeth twice daily and yet they had poor periodontal condition, it is possible that the children were giving socially desirable responses or that they had not acquired the proper brushing technique. It is recommended that more attention should be paid to proper toothbrushing in future oral health education programs for Guangdong children.

In the $1980 \mathrm{~s}$, the Chinese government banned the use of tetracycline in children (Editorial Board of Chinese Annals of Public Health, 1983), so it was expected that the prevalence of tetracycline-stained teeth should be very low in the Chinese child population. This was found to be true for the 5- to 6-yearold urban children surveyed in this study but not for the rural children. The reason for such a difference between the rural and urban children was unknown. Since the development of tetracycline staining in the primary dentition was mainly due to the administration of tetracycline from 5 months in utero to the first year of life (Mello, 1967; Pindborg, 1982), the higher prevalence among the rural children might be due to a more common practice among the doctors working in rural areas of prescribing tetracycline for pregnant women, or that the banned use of tetracycline for babies was not strictly followed in the rural areas. The extent to which tetracycline is used in selfmedication and its availability in the market warrants further investigation. To prevent this esthetic dental problem among Chinese children, government authorities in the rural areas should educate the health workers and the people about the side-effects of tetracycline and reinforce the ban on the use of this antibiotic in children.

Similar to the findings of a recent survey conducted in Central China (Peng et al., 1997), results from the multivariate analysis showed that being a girl, living in the rural areas, and having a more recent dental visit were associated with a higher DMFT score. However, in the former survey, children with higher consumption of sugary drinks and foods were also found to have higher DMFT scores, while in the present study, no significant association was found. When the rates of consumption of sugary drinks and foods 
by the 12-year-olds in the 2 studies were compared, it was found that children in Southern China reported less sugary drink and food consumption than children in Central China. However, the validity of the information might be in doubt, since it was difficult to show whether the children did consume fewer sugary drinks and foods or were just giving socially desirable responses. This is always a problem when asking questions concerning diet. In both surveys, no significant association was found between parental education level and the children's caries experience.

In conclusion, the prevalence of dental caries among the 5- to 6year-olds in Guangdong Province, Southern China, was high. The prevalence was higher among rural than urban children. The need for caries treatment was substantial, and there is an urgent need for establishing caries-preventive activities for pre-school children. The prevalence of tetracycline-stained teeth among the 5- to 6-year-old rural children was unacceptable, and the ban on the use of tetracycline in children should be reinforced. The prevalence of dental caries among the 12-year-olds was low, but most of the decayed teeth remained untreated. Moreover, their periodontal condition was poor. The children's knowledge about gum disease and the use of fluoride was found to be low. Thus, more oral health education programs should be organized to improve the above situation, especially for the rural children. Furthermore, in view of the scarce resources that are available for dental care and the disease pattern, the oral health policy for children in Southern China should emphasize prevention and simple treatment. Establishment of a school-based integrated package, such as the one proposed by the World Health Organization (Pakhomov, 1999), which consists of urgent oral treatment, affordable community oral disease prevention, and atraumatic restorative treatment, will probably improve the current situation to a large extent.

\section{ACKNOWLEDGMENT}

The University of Hong Kong (CRCG) and the Research Grants Council of Hong Kong financially supported this study.

\section{REFERENCES}

Chan JCY, So FHC, Yu FSH (1997). Oral health survey on primary school children in Hong Kong. Hong Kong: Department of Health, Hong Kong SAR Government.

Douglass JM, Wei Y, Zhang BX, Tinanoff N (1995). Caries prevalence and patterns in 3-6-year-old Beijing children. Community Dent Oral Epidemiol 23:340-343.

Editorial Board of Chinese Annals of Public Health (1983). Chinese Annals of Public Health 1983. Beijing: People's Health Publishing House.

Editorial Board of Chinese Journal of Stomatology (1983). Further analysis of dental caries in Chinese people-a compilation of articles. Chin J Stomatol 18:123-126 (in Chinese).

Editorial Board of West China Journal of Stomatology (1988). An analysis of the prevalence of dental caries in certain areas of our country. West China J Stomatol 6:100-103 (in Chinese).

Fejerskov O, Baelum V, Luan WM, Manji F (1994). Caries prevalence in Africa and the People's Republic of China. Int Dent J 44:425-433.

Feng XP, Shu CB, Kamibara M, Negame S (1998). A comparative study for the permanent teeth decay between Shanghai and Osaka citizens. Shanghai Stomatol 7(1):33-36.

Holm AK (1990). Caries in the preschool child: international trends. J Dent 18:291-295.

Hu D, Liu D (1992). Trends of caries prevalence and experience in children in Chengdu City, West China, 1982-90. Community Dent Oral Epidemiol 20:308-309.

Li G, Wang C, Li J, Ai Y, Zhang Y (1994). Dental caries in Yanan schoolchildren aged 12 years: China. Community Dent Oral
Epidemiol 22:208.

Lin HC, Wong MCM, Wang ZJ, Lo ECM (2001). Oral health knowledge, attitudes, and practices of Chinese adults. $J$ Dent Res 80:1466-1470.

Lo EC, Holmgren CJ, Hu DY, Wan HC (1999). Dental caries status and treatment needs of 12-13 year-old children in Sichuan Province, Southwestern China. Community Dent Health 16:114-116.

Mello HS (1967). The mechanism of tetracycline staining in primary and permanent teeth. J Dent Child 34:478-487.

Ministry of Public Health (1987). A national epidemiological survey of dental caries and periodontal disease among school children. Beijing: People's Health Publishing House (in Chinese).

Murray JJ (1994). Comments on results reported at the Second International Conference 'Changes in Caries Prevalence'. Int Dent $J$ 44:457-458.

Pakhomov GN (1999). Future trends in oral health and disease. Int Dent $J$ 49:27-32.

Peng B, Petersen PE, Fan MW, Tai BJ (1997). Oral health status and oral health behaviour of 12-year-old urban schoolchildren in the People's Republic of China. Community Dent Health 14:238-244.

Petersen PE, Esheng Z (1998). Dental caries and oral health behaviour situation of children, mothers and schoolteachers in Wuhan, People's Republic of China. Int Dent J 48:210-216.

Petersen PE, Guang LX (1994). Dental caries prevalence in a group of schoolchildren in Wuhan City, PR China, 1993. Community Dent Oral Epidemiol 22:465-466.

Pindborg JJ (1982). Aetiology of developmental enamel defects not related to fluorosis. Int Dent $J$ 32:123-134.

Qiu ZF, Shan WM, Lou WH, Chang YP, Ding HL, Cai DQ (1996). A comparison of incidence of dental caries among primary and middle school pupils in Shanghai in 1982 and 1990. Chin J Stomatol 31:113116 (in Chinese).

Schwarz E, Lo ECM (1994). Dental health knowledge and attitude among the middle-aged and the elderly in Hong Kong. Community Dent Oral Epidemiol 22:358-363.

Schwarz E, Lo ECM (1995). Oral health and dental care in Hong Kong. Int Dent J45:169-176.

Schwarz E, Zhang HG, Wang ZJ, Lin HC, Lo ECM, Corbet EF, et al. (2001). An oral health survey in Southern China, 1997: background and methodology. J Dent Res 80:1453-1458.

Shi Y, Barmes D, Bratthall D, Leclercq MH (1992). WHO pathfinder caries survey in Beijing extended with data for prevalence of mutans streptococci. Int Dent J 42:31-36.

Wang ZJ, Shen Y, Schwarz E (1994). Dental caries prevalence of 6-14year-old children in Guangdong, China. Community Dent Oral Epidemiol 22:340-341.

Warnakulasuriya KA (1989). Prevalence of selected developmental dental anomalies in children, in Sri Lanka. J Dent Child 56:137-139.

Wei SH, Shi Y, Barmes DE (1986). Needs and implementation of preventive dentistry in China. Community Dent Oral Epidemiol 14:19-23.

Wei SHY, Holm AK, Tong LS, Yuen SW (1993). Dental caries prevalence and related factors in 5-year-old children in Hong Kong. Pediatr Dent 15:116-119.

Wong MC, Schwarz E, Lo EC (1997). Patterns of dental caries severity in Chinese kindergarten children. Community Dent Oral Epidemiol 25:343-347.

World Health Organization (1988). Oral health global indicators for 2000. Geneva: WHO.

World Health Organization (1997). Oral health surveys: basic methods. 4th ed. Geneva: WHO.

Wright FAC, Deng J, Shi ST (1989). The dental health status of 6 and 12 year-old Beijing schoolchildren in 1987. Community Dent Health 6:121-130.

Yue SL (1980). Preliminary analysis of dental caries in Chinese people. Chin J Stomatol 15:1-4 (in Chinese).

Zheng CL, Yang SJ, Wu Y (1986). An epidemiological survey on the caries prevalence of school children in Guangdong-a summary. $J$ School Health 7(3):58-59 (in Chinese). 